\title{
Desarrollo de competencias digitales a partir del uso de vídeos didácticos
}

\section{Digital competences from the use of educational videos}

\author{
BOLAÑO García, Matilde ${ }^{1}$ \\ CÁRDENAS García, Erika T. ${ }^{2}$ \\ URIBE Miranda, Cristhian ${ }^{3}$
}

\begin{abstract}
Resumen
Las exigencias educativas requieren del desarrollo de ciertas competencias digitales. Desde esta perspectiva se propuso evaluar el uso de los vídeos didácticos para el fortalecimiento de las competencias digitales en los estudiantes de media técnica de la ciudad de Santa Marta, Colombia. Metodológicamente se orientó con un enfoque positivista, cuantitativo con diseño cuasiexperimental. Como resultado el grupo experimental mejoró el nivel de desarrollo cognoscitivo, mientras que el control no presentó mejoría significativa. Concluyendo la efectividad del uso de video didácticos.

Palabras clave: video, competencia, didáctica, educación, digital
\end{abstract}

\begin{abstract}
Educational demands require the development of certain digital skills. From this perspective, it was proposed to evaluate the use of didactic videos to strengthen digital skills in technical media students in the city of Santa Marta, Colombia. Methodologically, it was oriented with a positivist, quantitative approach with a quasi-experimental design. As a result, the experimental group improved the level of cognitive development, while the control group did not show significant improvement. Concluding the effectiveness of the use of educational video.

Key words: video, competition, didactics, education, digital
\end{abstract}

\section{Introducción}

Los avances tecnológicos han impulsado nuevos espacios donde es necesario integrar recursos tecnológicos que permiten consolidar los procesos de aprendizaje de manera más rápida y efectiva. Al respecto, Robles y Zambrano (2020) aseguran que las nuevas tecnologías mejoran los ambientes didácticos y metodológicos, con un buena gestión y clima dentro del aula, para que el alumno optimice su aprendizaje. Además, afirman son necesarias para que se logren oportunidades, consiguiendo acceso a la innovación y aportando nuevas

\footnotetext{
${ }^{1}$ Doctora en Ciencias de la Educación, Magíster a informática educativa. Docente e investigadora de educación superior. Universidad del Magdalena. Santa Marta, Colombia. Correo electrónico: mbolano@unimagdalena.edu.co. https://orcid.org/0000-0002-5514-2992

2 Estudiante de Licenciatura en informática. Universidad del Magdalena, Santa Marta, Colombia. Correo electrónico: cardenaserikatatiana@gmail.com ORCID: 0000-0001-5529-9335

${ }^{3}$ Licenciado en educación básica con énfasis en informática. Universidad del Magdalena, Santa Marta, Colombia. Correo electrónico: cristianuribem7@gmail.com ORCID: 0000-0001-7593-4412
} 
experiencias. Otras técnicas aplicadas son: el círculo de aprendizaje y las expediciones pedagógicas siendo necesarias para que Latinoamérica continúe su desarrollo educativo.

Al dar una mirada a los sistemas educativos de América Latina, desde finales del siglo XX, países como Uruguay, Chile y Colombia, ya reportaban valiosas experiencias en la aplicación de herramientas tecnológicas en el contexto educativo. En este sentido, Vargas (2020) sostiene que en América Latina y el Caribe el siglo XXI se configura como la era donde las innovaciones tecnológicas abarcan todos los ámbitos en los que se desarrolla la sociedad, tales como la filosofía, política, ciencias sociales, ciencias básicas, entre otros. Al respecto, la educación tecnocientífica (ETCS) es conocida como el conjunto de disciplinas que interactúan para estudiar los aspectos sociales de la ciencia y la tecnología con relación al ámbito educativo en pro de la sociedad. En este sentido, existen organizaciones que promueven una educación para todos como un derecho, tal como lo establece la UNESCO (1997).

Para Perdomo, Martínez \& Barreto (2020), en la competencia digital de los estudiantes latinoamericanos predominan los acercamientos descriptivos y de corto alcance en cuanto a las poblaciones estudiadas. Los autores aseguran es escasa la investigación dirigida a evaluar la competencia y, cuando se hace, se emplean instrumentos diseñados debido a la ausencia de estándares propios. Márquez et al. (2020) señala que los países latinoamericanos procesan información discontinua y no lineal; acceden a la información principalmente a partir de fuentes digitales (imágenes en movimiento, música, por encima del texto); están acostumbrados a procesos de aprendizaje directos que ofrecen retroalimentación inmediata, rápidos, concretos y repetitivos; toman decisiones en contextos digitales muy bien diseñados y excitantes (sobre todo si se trata de juegos); crean materiales originales para la web, ya sea a través de su blog o de su página personal, con fotografías, videos o imágenes originales.

En ese sentido, Orellana y Pósito (2019) rescatan su eficacia para activar el aprendizaje a través de estímulos a los órganos sensoriales de manera conjunta, específicamente vista y oído, involucrando varios espectros del aprendizaje, con lo cual los videos didácticos logran captar mejor la atención de los estudiantes independientemente de su estilo de aprendizaje y, en consecuencia, mejorar las probabilidades de éxito de los fines educativos. Adicionalmente, según Bernal-Torres, et al. (2020) la mayoría de los docentes considera importante producir un video didáctico para utilizarlo en clase, y que existen aspectos importantes que se evidencian en un tema cuando se quiere profundizar, puesto que los estudiantes reconocerían el valor del lenguaje «extra verbal» y atraería su atención en gran medida.

Al respecto, Troncoso-Pantoja, et al. (2019) señalan que los videos didácticos son un aporte a la formación profesional de los estudiantes, al actuar como un canal facilitador en la entrega de contenidos, al favorecer el aprendizaje activo e incrementar la creatividad del docente y su forma de comunicar las temáticas disciplinares. El uso de los videos como estrategia de formación pedagógica y herramienta educativa es reconocida y utilizada en el proceso de enseñanza y aprendizaje de estudiantes. Orellana y Pósito (2019), al referirse a los videos didácticos, consideran que el uso de los órganos sensoriales en conjunto, por ejemplo vista y oído, contribuyen mucho más al proceso de aprendizaje que si solo se escucharan o miraran contenidos sin sonidos.

La autora anteriormente mencionada, citando a Vigotski (1977), afirma que los órganos sensoriales controlan los mayores estímulos, entre los cuales está la vista, con un $83 \%$, seguido del oído con $11 \%$ y finalmente, en menor grado, el kinestésico. Todos ellos son los llamados canales perceptivos, siendo el video didáctico uno de esos medios que permiten involucrar a los alumnos y activar el aprendizaje emocional y cognitivo.

No obstante, es importante destacar que su implementación no garantiza la adquisición de competencias en los estudiantes, es decir, es necesario considerar que la interacción con los videos didácticos es uno de los elementos en la ecuación, pero dependerá del grado de compromiso e interés, materiales adicionales y las 
actividades complementarias lo que enriquecerán el proceso como un todo, Kovacs (2016) citado por Orellana y Pósito (2019).

Para Troncoso, et al. (2019) el uso de los videos como herramienta educativa es conocida y utilizada en el proceso de enseñanza y aprendizaje en distintas disciplinas, reconociendo que puede considerarse como un recurso que favorece la adquisición de conocimientos sobre una determinada temática, un preferible desempeño operativo y una mayor satisfacción por parte de los estudiantes al interactuar con el contenido.

La premisa principal al evaluar el uso de los vídeos didácticos para fortalecer las competencias digitales, se basa en creer que cuando el estudiante tiene la posibilidad de controlar la velocidad en que los contenidos son administrados, es decir parar, adelantar y controlar el ritmo de la información, es posible aprender más y mejor Linarez (2015)

Al revisar experiencias del uso en varios escenarios es posible encontrar un prolífero abanico de trabajos de investigación con poblaciones que van desde la educación preescolar hasta la universitaria. En ese sentido, conviene destacar a Espinoza-Freire (2020), al afirmar en su trabajo de investigación "Políticas educativas de integración de las tecnologías de la información y las comunicaciones en el sistema educativo" la importancia de generar un espacio de reflexión que propicie las políticas educativas y su estrecha relación entre el entorno escolar, la formación integral, la salud del estudiante y todo el acompañamiento del desarrollo de competencias profesionales que adquiera durante su proceso formativo.

Al respecto, la Oficina de Innovación Educativa perteneciente al Ministerio de Educacional Nacional de Colombia (2008), manifiesta la importancia de emplear las políticas para el desarrollo de competencias apoyados por la tecnologías, en este sentido ha trazado unos lineamientos para trabajar las tecnologías en el sistema educativo, convirtiéndose en una herramienta indispensable para la formación integral no solo en la población regular sino también para población no regular, en la que dichas habilidades le ofrecen un sinnúmero de posibilidades para avanzar hacia la autonomía, con el uso videos didácticos de corta duración, como píldoras informativas podrían ser más efectivas en el aprendizaje, Bolaño (2019).

De hecho, plataformas como Youtube han demostrado la amplia diversidad de temas y contenidos que pueden ser utilizados tanto en la educación formal como en la no formal (Bolaño, 2017; González, 2018). De acuerdo con Alvarado, Dehesa, López y Márquez (2017) las plataformas tecnológicas de hoy en día ofrecen una alternativa accesible, pero destacan que es importante analizar en los contenidos que se ofrecen, particularmente aquellos que brindan mayor cantidad de información específica y, consecuentemente, acompañada de imágenes, sonidos y lecturas acordes a la profundidad o nivel esperado. El presente artículo se propuso evaluar el uso de los vídeos didácticos para el desarrollo de competencias digitales en los estudiantes de media técnica de la ciudad de Santa Marta, Colombia.

\subsection{Competencias Digitales (CD)}

Los complejos procesos educativos de un mundo globalizado exigen el desarrollo de ciertas competencias, además de las básicas que ha ofrecido la escuela desde tiempos remotos, y que han ido evolucionando de acuerdo a las exigencias de las sociedades. Las competencias digitales se conciben como la habilidad de conocer, emplear y valorar el uso de herramientas tecnológicas para la búsqueda de información, la comunicación no presencial y la creación de conocimiento con el uso del computador. Gálvez (2017).

La anterior definición es complementada por Velázquez, \& Cupil, (2020) al señalar que las competencias digitales contribuyen a que el estudiante sea competente y esté a la vanguardia de la educación, debe ser capaz de resolver problemas, poseer diferentes habilidades tecnológicas de manera que sepa manipular los recursos 
tecnológicos, maneje y procese información confiable y de calidad. Estas competencias redefinirán su perfil y el estudiante tendrá una formación integral, que se constituirá con diversos saberes.

De acuerdo con Levano-Francia et al. (2019), las competencias digitales han generado diversas líneas de investigación y nuevos avances en el uso de las TIC. Su vasta trascendencia en el ámbito de su aplicación a la tecnología educativa, cuyo espectro de acción abarca diversas proyecciones, tanto de aprendizajes e investigación como recreacionales y sociales. Al respecto Arango et al. (2020) aseguran que las competencias digitales posibilitan el acercamiento al tratamiento de la información, a una mejor comunicación, permitiendo emplear estrategias de participación y aprendizaje en equipo.

Adicionalmente, Leal (2020) citando a INTEF (2017), definen a la competencia digital como el uso crítico y seguro de las tecnologías para el trabajo y el tiempo libre, la comunicación, en las que apoyados por las habilidades básicas podrán recuperar, evaluar, almacenar, producir, presentar e interactuar con la información para comunicar y participar en redes de comunicación a través de internet, la cual se covierte en una de las competencias claves que deben desarrollar los ciudadanos con el fin de aprovechar las TIC para el aprendizaje permanente y la participación en la sociedad del conocimiento. Cejas et al. (2020), citando a Zúñiga (2016), afirman que las competencias digitales son elementos que contribuyen significativamente al desarrollo del autoaprendizaje y, en gran medida, son también herramientas que permiten aumentar el capital de conocimiento científico, promoviendo el uso efectivo y eficiente de los recursos digitales.

En torno a lo expuesto, Bolaño (2019) destaca la importancia del uso de las competencias digitales argumentando que el auge de las TIC ha generado diversas herramientas, productos y recursos que permiten acceder y constatar la información, tanto dentro como fuera del aula, proponiendo alternativas y permitiendo alcanzar altos niveles de autonomía, que le abren amplias posibilidades de inclusión social, pedagógica y laboral a los educandos.

Los anteriores autores coinciden al plantear que la importancia de las CD se centra en desarrollar las capacidades, destrezas y habilidades del estudiantado con manejo de tecnologías que favorecen el aprendizaje, su interacción con el contexto que lo rodea y su interrelación con otros y aprender colaborativamente o de manera autónoma, en la que se debe conocer el procedimiento y actitudes para su aplicación, de allí la relevancia de promover su desarrollo en todos los niveles del sistema educativo y posteriormente, la evaluación del logro de dichas habilidades a luz de las herramientas disponibles.

\subsection{Competencias Digitales Cognitivas (CDC)}

Las competencias digitales cognitivas comprenden el conocimiento (saber) de los elementos físicos y los no tangibles del computador, a estos elementos se le denominan componentes básicos. Tal competencia es alcanzada cuando la persona es capaz de identificar los elementos externos de un equipo, las funciones de cada uno de los dispositivos periféricos que le componen, conoce el paquete de office y los programas que integran el computador y con el paso del tiempo, se ha incluido el conocimiento del uso de internet (Instituto Nacional de Evaluación Educativa, 2016).

No obstante, Mañas \& Roig-Vila (2019) afirman que las TIC juegan un papel importante en el contexto de la sociedad actual, al ayudar en la adquisición de competencias a partir del autoaprendizaje y la interacción eficientemente con el uso del computador en la elaboración de tareas ya sea en el contexto laboral o profesional. Según los anteriores autores, esto implica que el desarrollo de la competencia digital cognitiva conlleva a un proceso de reflexión en los que generan preguntas ¿cómo aprendo?, ¿cómo aplico eficientemente mi conocimiento?, entre otras. Es decir, intrínsicamente los procesos superiores del pensamiento entran en juego para enriquecer la experiencia de aprendizaje. 
Finalmente, Nieto \& De la Rosa (2019) afirman que la competencia cognositiva consiste en el conjunto de conocimientos y habilidades para buscar, seleccionar, analizar, interpretar y recrear la gran cantidad de información a la que se tiene acceso a partir de las tecnologías digitales y dentro de la sociedad de la información. Adicionalmente Nieto \& De la Rosa (2019) señalan que las competencias digitales cognitivas están relacionadas con la formación del sujeto y van a permitirle el conocimiento sobre los contextos tecnológicos en los que se encuentra, proporcionándole poder reflexionar, desarrollar habilidades y destrezas para el adecuado manejo de los equipos y programas digitales, ampliando el saber y el conocer de lo que le ofrecen las tecnologías de la información y comunicación.

En conclusión, podría afirmarse que las competencias digitales cognitivas se refieren al desarrollo del saber y los conocimientos, tanto de los elementos básicos que conforman un equipo de computación (hardware) como los programas y sistemas operativos que lo integran y el manejo del internet.

\subsection{Competencias Digitales Procedimentales (CDP)}

Sobre las competencias digitales procedimentales, Benítez (2020), citando a Deursen, Dijk y Peters (2017), afirman que son las que desarrolla el individuo en el uso de los equipos electrónicos como el computador, saberes procedimentales para orientarlos en las búsquedas, la selección de información y para transmitir contenidos sustantivos de cada materia. Por su parte, Morales-Rocha et al. (2020), consideran que las competencias procedimentales constituyen un referente metodológico, es un tipo de saber que conduce al saber hacer. Es decir, se refieren a acciones y estrategias que permiten resolver problemas, producir hechos u objetos y lograr conseguir objetivos.

Luego del análisis anterior, es posible deducir que competencias procedimentales constituyen un referente metodológico y de procedimiento. Suponen una combinación de habilidades, manuales y capacidades cognitivas que posibilitan la competencia profesional, en este sentido incluyen destrezas en manipular ideas y el contexto en el que se desenvuelven los estudiantes, destreza física. Por tanto, son las encargadas de dotar a las personas de los diversos métodos y habilidades que le permitirán ser un profesional competente, en este caso, favorecerán el desarrollo de destrezas que podrán ser empleadas en los equipos computarizados, y en el uso constante de la tecnología.

\subsection{Competencias Digitales Reflexivas y Actitudinales (CDRA)}

Partiendo de lo definido por Morales et al (2020) las competencias reflexivas y actitudinales están orientadas hacia el saber ser, al convertir las actitudes y los valores en contenidos educativos, define normas a seguir. Los contenidos actitudinales no están limitados a una disciplina en particular, ya que en todas las disciplinas se consideran valores como la libertad, la verdad, la solidaridad, la justicia, la igualdad, entre otros. Estos contenidos hacen referencia al aspecto valorativo que todo conocimiento tiene, y consecuentemente al compromiso personal y social que implica el saber.

Al consultar a Gálvez \& Tolaha (2019) definen las competencias reflexivas y actitudinales, como esos conocimientos y destrezas que desarrolla el estudiante para comprender, transformar y participar en el contexto en el que se desenvuelve. Éstas se relacionan directamente con el "saber-ser" o "saber-actuar" frente a una situación determinada. Por esta razón son competencias extremadamente útiles para el desempeño profesional y sin las cuales muchos de los conocimientos adquiridos no podrían ser aprovechados.

Gálvez \& Tolaha (2019) afirman que entre las competencias fundamentales destaca las actitudinales y reflexivas, como son las habilidades para hablar y escribir de manera fluida y con calidad, trabajar en equipo, aprender por sí mismo, igualmente la búsqueda de información, reflexionar sobre el aprendizaje alcanzado y planificar las actividades a realizar, resolver los problemas cotidianos, desarrollar la iniciativa y creatividad. Luego de la 
revisión de lo anteriormente expuesto se observan similitudes en las definiciones sobre las Competencias digitales reflexivas actitudinales (CDRA), al considerarlas como habilidades que presentan los sujetos partiendo de sus intereses y sentimientos. De esta manera, las competencias digitales reflexivas actitudinales no son más que aquellas habilidades que van a permitir a un individuo poner de manifiesto sentimientos y emociones.

Una vez definido los conceptos claves en torno a las competencias a estudiar en el presente artículo, a modo de resumen la tabla 1 presenta cómo son entendidas las competencias en el experimento que se planteará a continuación y detallan los aspectos que se definen cada competencia.

Tabla 1

Tipificación de las competencias digitales.

\begin{tabular}{|c|c|c|}
\hline CD Cognitiva & CD Procedimental & CD Reflexiva y Actitudinal \\
\hline $\begin{array}{l}\text { Son aquellos que hacen referencia a la } \\
\text { comprensión y conocimiento de las } \\
\text { fuentes de información bien sean } \\
\text { primarias y secundarias intrínsecas al } \\
\text { proceso de búsqueda. Implican aprender } \\
\text { a aprender a lo largo de la vida, } \\
\text { abarcando: conceptos, haciendo un buen } \\
\text { uso de las TIC y participación a través de } \\
\text { ellas } \\
\text { Ejemplo: qué es un artículo, conocer los } \\
\text { tipos de artículos, entre otros. } \\
\text { (Saber conceptos y datos)-Qué }\end{array}$ & $\begin{array}{l}\text { Son aquellos que permiten dejar en } \\
\text { manifiesto el hacer, la parte } \\
\text { práctica. Implican seleccionar, } \\
\text { sistematizar y emplear la } \\
\text { información teniendo en cuenta la } \\
\text { construcción del conocimiento } \\
\text { Ejemplo: cómo citar una referencia, } \\
\text { emplear motores de búsqueda } \\
\text { eficientes de la información. } \\
\text { (Saber proceso y procedimientos) - } \\
\text { Cómo }\end{array}$ & $\begin{array}{l}\text { Son aquellas que permiten } \\
\text { establecer criterios de } \\
\text { selección de información y } \\
\text { validación. Implican las } \\
\text { habilidades actitudinales, lo } \\
\text { discrimina en } 3 \text { campos } \\
\text { manejo de situaciones, } \\
\text { competencias para la } \\
\text { convivencia y competencias } \\
\text { para la vida en sociedad } \\
\text { Ejemplo, identificar cuál } \\
\text { información es plagio y cuál } \\
\text { no y evitarlo. } \\
\text { (Actitud y reflexión) }\end{array}$ \\
\hline
\end{tabular}

Fuente: Elaboración propia, basado en Lizana (2018) y Paz (2018)

\section{Metodología}

La presente investigación se desarrolló atendiendo los diversos procedimientos de una investigación cuantitativa, de campo de acuerdo con Benitez (2020) citando a Fernández y Fernández (2016) son aquellos estudios, datos de manera directa a los hechos donde ocurren, en este caso la institución educativa técnica industrial. En cuanto a su diseño, se enmarca en la tipología cuasi experimental teniendo como objetivo determinar las competencias digitales de treinta y ocho (38) estudiantes del grado décimo, jornada de la mañana de la Institución Educativa Distrital Técnico Industrial de Santa Marta, Colombia, durante el año 2018, con los cuales se conformaron dos grupos (control y experimental) de diecinueve (19) sujetos cada uno, pertenecientes a la misma sección. El criterio de selección de los 19 sujetos para cada grupo: Cursar Tecnología e Informática, en el décimo grado, jornada de la mañana de la Institución Educativa estudiada, de tal manera que se garantizaran las condiciones de un cuasi experimento que permitiera corroborar o negar la hipótesis planteada.

Por tanto, se trabajó con un grupo ya conformado. En palabras de Fernández y Fernández (2016) es posible que los grupos sean heterogéneos en sus características, y que frente a su tratamiento aplicado o la variable independiente estas puedan afectar la posibilidad en sus respuestas de manera consecuente. En este caso, dos grupos de estudiantes, uno que empleó las guías para medir el uso de videos digitales para el fortalecimiento de la competencias digital y el otro, continuó recibiendo clases tradicionales.

Como técnica de recolección de datos se empleó la observación mediante encuesta, es decir, a través de un cuestionario tipo prueba de conocimiento para medir las competencias digitales presentes en los estudiantes, antes y después del experimento. El instrumento de elaboración propia, estuvo conformado por dieciocho (18) 
ítems cerrados, que fueron divididos en tres (3) secciones, uno para cada competencia, con dos (2) tipos de opciones de respuestas, a saber: verdadero y falso y selección múltiple.

Antes de su aplicación definitiva, la prueba de conocimiento fue sometida a la revisión de cinco expertos tanto en contenido, como en metodología, quienes determinaron la validez de criterio y objetividad del instrumento. Según Kerlinger y Lee (1999), este paso proporciona el grado de validez en el que un instrumento es capaz de producir resultados consistentes y coherentes, además de proporcionar datos relevantes sobre la variable objeto de estudio en función a los aspectos teóricos que interesan estudiar.

Para determinar la confiabilidad del instrumento de investigación, se aplicó la prueba a una muestra piloto de diez (10) estudiantes con características similares a los del experimento en cuestión. A los datos obtenidos se les calculó el Coeficiente Kuder Richardson (KR 20) para ítems continuos, con ello se determinó el nivel de confiabilidad que proporcionaba el instrumento. Los resultados evidenciaron una confiabilidad de 0.72 , por lo que se puede decir que su administración es altamente confiable, según los criterios de Ruiz (2007).

Para llevar a cabo la investigación fue necesario diseñar un plan instruccional de 5 horas semanales por 4 semanas, para aplicar el uso de videos didácticos y de esta manera se planteó la siguiente hipótesi de trabajo:

H1: El uso de vídeos didácticos es efectivo para fortalecer las CD.

La investigación se realizó en 3 fases denominadas 1. preparación, 2.diseño y c3aplicación. Se describen a continuación:

1. Preparación, los investigadores realizaron el levantamiento teórico de la información, definieron las variables de estudios, métodos e instrumentos de recolección de datos, realizaron las gestiones institucionales para llevar a cabo el experimento con la población antes descrita

2. Diseño, para este momento se elaboró el plan de actividades, el diseño metodológico de la investigación y sus instrumentos de recolección de datos, pruebas de validez y confiabilidad y realizaron las solicitudes de consentimiento informado a los padres de los estudiantes participantes;

3. Aplicación, se desarrolló el plan de actividades con ambos grupos y se aplicó el instrumento. Los estudiantes contaron con 30 minutos para contestar la prueba.

En la prueba se abordaron contenidos enfocados a las fases de fundamentación, utilización, creación y compartir información, como:

- Internet y navegadores

- Ventanas y la organización de la información

- Técnica y estrategias de búsqueda de información

- Uso y búsqueda eficiente de la información

- Herramientas de información sincrónica y asincrónica

- $\quad$ Sistemas de gestión de contenidos

- $\quad$ Las normas de Netiqueta

- El plagio

Finalmente, se llevó a cabo el análisis de resultados y elaboración de informes. Luego del registro de la información de las diferentes fases, se tabularon y analizaron los resultados que arrojaron los instrumentos de recolección de datos para construir los informes que de manera resumida presentaron los hallazgos y logros de la investigación.

Los datos recolectados durante el experimento fueron tabulados utilizando el programa Microsoft Office Excel 2013 y el paquete estadístico SPSS versión 21. Para tabularlos, a cada una de las respuestas correctas se les 
asignó el valor uno (1) y a las incorrectas, cero (0). Como resultado, a cada indicador y dimensión de la variable se le calculó la media para determinar el logro de las competencias esperadas, a partir de lo cual se realizaron los estadísticos descriptivos e inferenciales, que permitieron comparar los resultados obtenidos entre los grupos (control y experimental), particularmente, la existencia de diferencias significativas en las medias de cada grupo, a fin de comprobar la hipótesis de la investigación. Los resultados obtenidos se organizaron en tablas y gráficos para su posterior interpretación conforme al baremo (tabla 2).

Tabla 2

Baremo de conversión

\begin{tabular}{|c|c|}
\hline Intervalos & Categorías \\
\hline $0,00-0,16$ & Muy bajo desarrollo \\
\hline $0,17-0,33$ & Bajo desarrollo \\
\hline $0,34-0,50$ & Medio bajo desarrollo \\
\hline $0,51-0,67$ & Medio alto desarrollo \\
\hline $0,68-0,84$ & Alto desarrollo \\
\hline $0,85-1,00$ & Muy alto desarrollo \\
\hline
\end{tabular}

Fuente: elaboración propia

\section{Resultados}

La relevancia del uso de videos didácticos en la práctica educativa actual es indudable, por tanto, contar con un adecuado nivel de competencias digitales es fundamental para que la incorporación de la tecnología pueda ser armónica con el proceso educativo. Por tal razón, en primera instancia, se aplicó la prueba de conocimiento en forma de pretest para ambos grupos y así determinar el estado inicial de estas competencias digitales en los estudiantes.

Como puede observarse en la figura 1, la mayoría de las respuestas dadas en la prueba de conocimiento aplicada al grupo control resultaron incorrectas o negativas, particularmente, en las preguntas referidas a las competencias digitales cognitivas (78 \%) y competencias digitales procedimentales (73\%), lo que indica una dificultad para afrontar las tareas relacionadas con dichas competencias, en menor proporción (63 \%) pero igualmente desfavorable, la mayoría de las respuestas para las competencias digitales reflexivas y actitudinales resultaron negativas. Es decir, conocen poco, trabajan poco y reconocen escasos valores de las competencias digitales.

Figura 1

Competencias digitales

pre test grupo control

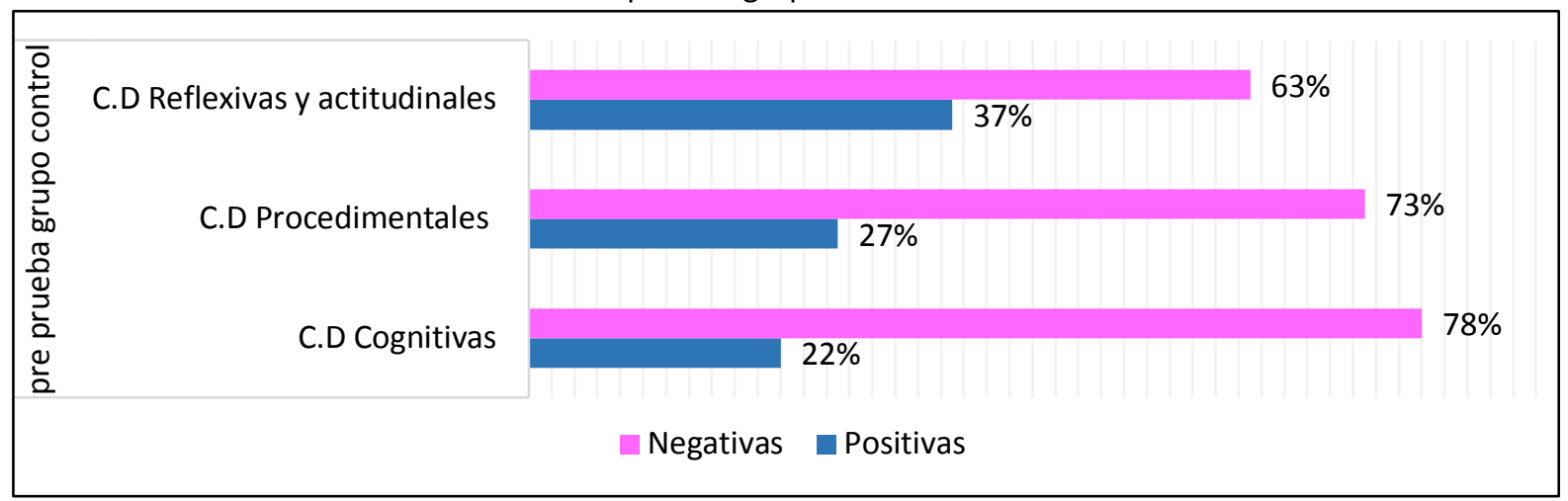

Fuente: Elaboración propia 
Al aplicar el mismo instrumento al grupo experimental, la figura 2 muestra que la situación es similar al grupo control, debido a que la mayoría de los estudiantes no acertaron las respuestas del instrumento, lo cual implica una tendencia negativa en sus precisiones sobre las competencias digitales, de hecho este grupo, al igual que el control, mostró mayores niveles de respuestas negativas en lo respectivo a competencias digitales cognitivas (79 \%) y competencias digitales procedimentales (73\%) aunque fue ligeramente más alto el nivel de respuestas negativas para las competencias digitales reflexivas y actitudinales $(67 \%)$ con respecto al grupo control. Es decir, poco saben, aplican y actúan en el campo de las competencias digitales.

Figura 2

Competencias digitales pretest grupo Experimental
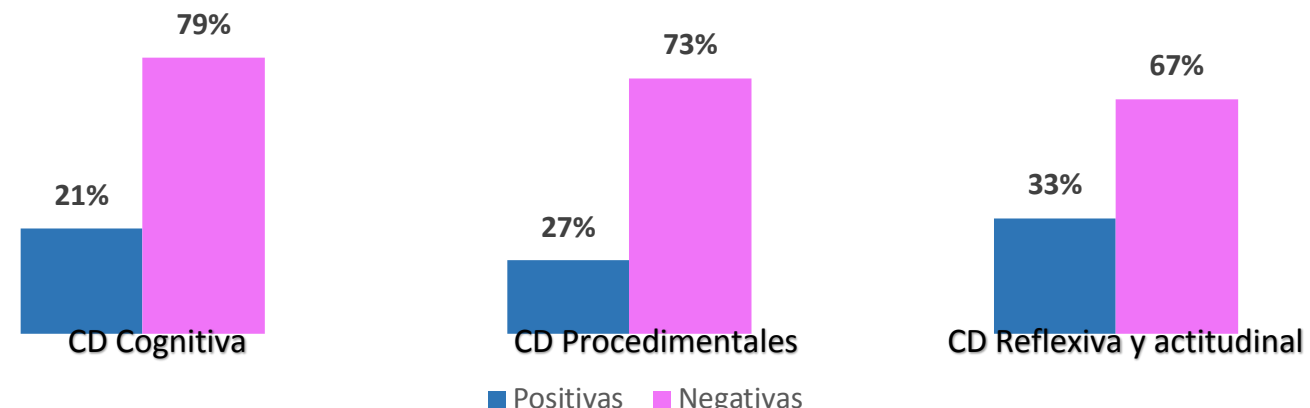

Fuente: elaboración propia

Aparentemente ambos grupos se encuentran en condiciones similares, lo cual sería el estado ideal para iniciar el experimento con uso de videos digitales para el fortalecimento de las competencias digitales, esta información además proporciona a los investigadores un diagnóstico del estado actual de dichas competencias y las áreas de énfasis a desarrollar con el plan de actividades. No obstante, para validar estadísticamente la suposición de que ambos grupos se encuentran en similares condiciones, se realizó una prueba de hipótesis (tabla 3) a través de la T de Student con el fin de comparar las medias aritméticas obtenidas por los grupos, en este caso, tomando en cuenta todas las respuestas del cuestionario.

Tabla 3

Prueba T de Student del pre test para grupo experimental y control.

\begin{tabular}{|l|r|r|}
\hline & Variable 1 & Variable 2 \\
Vedia & 5,15 & 5,05 \\
Observacionza & 0,55526316 & 0,365789474 \\
Diferencia hipotética de las medias & 19 & 19 \\
Grados de libertad & 0 & 18 \\
Estadístico t & 0,69798244 & \\
$\mathrm{P}(\mathrm{T}<=\mathrm{t})$ una cola & 0,24682129 & \\
Valor crítico de t (una cola) & 1,72913279 & \\
$\mathrm{P}(\mathrm{T}<=\mathrm{t})$ dos colas & 0,49364259 & \\
Valor crítico de $t$ (dos colas) & 2,09302405 & \\
\hline
\end{tabular}

Fuente: elaboración propia 
De acuerdo con los resultados de la tabla anterior, se observa que el valor de la T calculada fue de 0,697, que al compararla con la T tabulada o valor crítico de las T 2,09 (dos colas), con 18 grados de libertad (n-1), evidenciándose no existir discrepancias significativas entre las medias correspondientes a ambos grupos, se concluye que ambos grupos se encuentran en igualdad de condiciones.

La fase de experimentación se fundamentó en el desarrollo de un diseño instruccional para el uso de videos didácticos, con una duración de 20 horas mientras los estudiantes del grupo control recibieron clases tradicionales en el mismo período de tiempo. Cumplida la fase de experimentación desarrollada durante 4 semanas se procedió nuevamente a la aplicación de la prueba de conocimiento tanto para el grupo control como el experimental, en la figura 3 se muestran los resultados del postest del grupo control, a quienes no se les aplicó un estímulo diferente a las clases tradicionales. Como se puede apreciar en la figura 3 hubo una ligera mejoría en el porcentaje de respuestas positivas o correctas en el cuestionario, sin embargo, persisten altas concentraciones de respuestas negativas en los tres indicadores explorados, particularmente en las competencias digitales procedimentales (72\%), esto implica que, aunque tienen mejor nivel de conocimiento, su capacidad para demostrar el saber hacer se encuentra limitada.

Figura 3

Competencias digitales post prueba grupo control

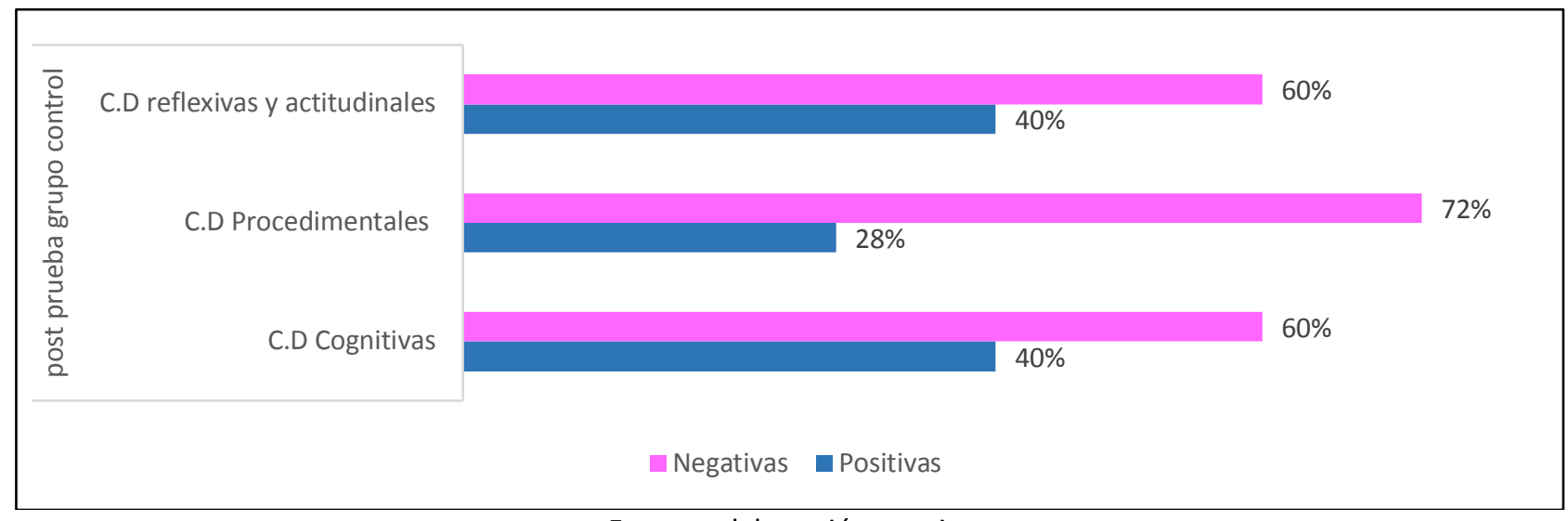

Fuente: elaboración propia.

A diferencia del grupo control, el experimental contó con un plan de actividades y desarrollo instruccional intencionalmente diseñado para promover el desarrollo de las competencias digitales a través del uso de videos didácticos. La figura 4, evidencia los resultados luego de la aplicación de la prueba de conocimiento después del experimento, encontrando que, en primer lugar, se destaca el significativo aumento en el desarrollo cognitivo de las competencias digitales cognitivas (74\%), es decir, ahora conocen teóricamente los aspectos del saber referido a esta competencia, mientras que en la competencia digital procedimental se movilizó en ambos sentidos, bajó la cantidad de respuestas negativas a 54 \% y aumentó las respuestas positivas o correctas (46 \%), es decir, mayor dominio en el hacer con tecnología. Finalmente, en la competencia digital reflexiva y actitudinal, lo más relevante a destacar es que se ubicaron en un $50 \%$ las respuestas positivas y negativas, si bien es una mejoría, no aumentó significativamente y considerando su relevancia en la consolidación de las competencias digitales en diferentes ámbitos de la vida académica y profesional. 
Figura 4

Competencias digitales post

prueba grupo experimental
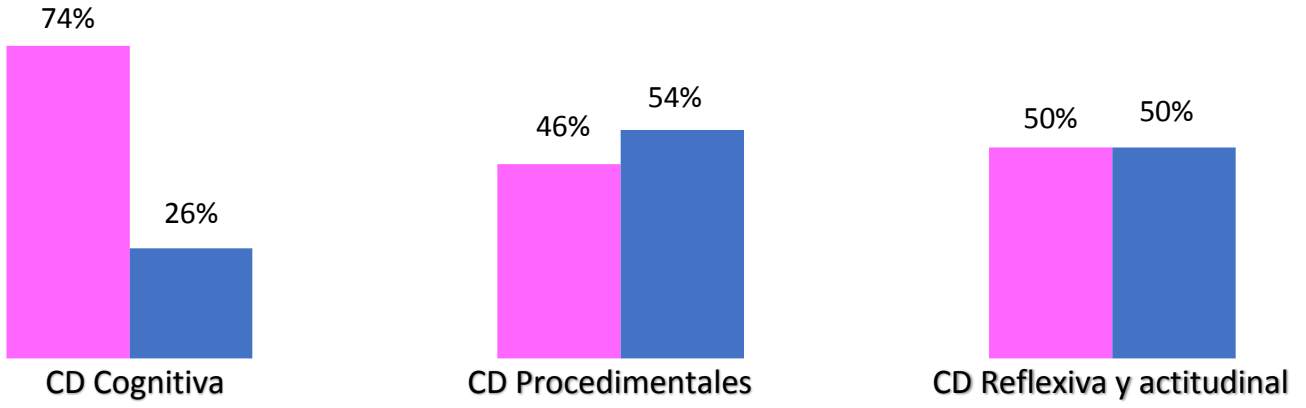

Fuente: Elaboración propia

A la luz de los anteriores resultados, se aprecia que porcentualmente el desempeño del grupo experimental en cada uno de los indicadores analizados fue mejor que la del grupo control, en términos de la cantidad de respuestas correctas o positivas alcanzadas en el postest. No obstante, nuevamente, se acude al estadístico T de Student (tabla 4) para corroborar las diferencias de medias y así comprobar o rechazar la hipótesis planteada.

Tabla 4

Prueba t para post prueba para

grupo control y experimental.

\begin{tabular}{|lrr|}
\hline & Variable 1 & Variable 2 \\
Vedia & 10,1 & 6,55 \\
Observaciones & 2,93684211 & 1,839473684 \\
Diferencia hipotética de las medias & 19 & 19 \\
Grados de libertad & 0 & \\
Estadístico t & 18 & \\
$P(T<=t)$ una cola & 14,4453745 & \\
Valor crítico de t (una cola) & $5,3291 \mathrm{E}-12$ & \\
$P(T<=t)$ dos colas & 1,72913279 & \\
Valor crítico de t (dos colas) & $1,0658 \mathrm{E}-11$ & \\
\hline
\end{tabular}

Fuente: elaboración propia

Luego de la aplicación del diseño instruccional y experimental sobre el desarrollo de competencias digitales al grupo experimental y comparar dichos resultados con el grupo control (a quienes no se les aplicó el diseño), se aplicó la T Student para establecer las posibles diferencias que pudieran existir entre las medias de cada grupo, con lo que se puede considerar que si hay diferencias significativas en función de que la T calculada fue de 14,44 mientras que la $T$ tabulada con 18 grados de libertad fue de 2,09, rechazando entonces la premisa de la igualdad de medias. Es decir, al ser estadísticamente diferentes las medias encontradas se asume como cierta la hipótesis de que el uso de vídeos didácticos es efectivo para el desarrollo de CD.

\subsection{Discusión}

Luego de analizar los resultados de la investigación, y al contrastarlos con los postulados teóricos de los autores consultados, se considera que las competencias digitales durante el período del pre test para ambos grupos, contradicen lo expuesto por Arango, et al. (2020), quienes plantean que las CD posibilitan el acercamiento al tratamiento de la información, a una mejor comunicación, permitiendo emplear estrategias de participación y 
aprendizaje en equipo, las mismas van más allá del conocimiento, porque permiten la utilización correcta de las tecnologías, como en el caso del manejo de los videos didácticos para fortalecer dichas competencias. Los resultados apuntan hacia la necesidad el deber desarrollar unas acciones para fortalecer CD.

Tabla 5

Desarrollo de los tipos de competencias digitales (pre prueba).

\begin{tabular}{|c|c|c|c|}
\hline Indicadores & Cognoscitivos & Procedimentales & Reflexivas y actitudinales \\
\hline Grupo control & $\begin{array}{c}0,22 \\
\text { Bajo desarrollo }\end{array}$ & $\begin{array}{c}0,28 \\
\text { Bajo desarrollo }\end{array}$ & $\begin{array}{c}\text { Medio bajo desarrollo } \\
\text { Promedio de la dimensión }\end{array}$ \\
\hline Grupo experimental & $\begin{array}{c}0,32 \\
\text { Bajo desarrollo }\end{array}$ & $\begin{array}{c}0,29 \text { (bajo desarrollo) } \\
\text { Bajo desarrollo }\end{array}$ & $\begin{array}{c}0,32 \\
\text { Bajo desarrollo }\end{array}$ \\
\hline Promedio de la dimensión & \multicolumn{3}{|c|}{0,28 (bajo desarrollo) } \\
\hline
\end{tabular}

Fuente: elaboración propia

A esta altura del análisis es preciso comparar los grupos de manera consolidada, por tal razón, la tabla 5 consolida los resultados de la dimensión desarrollo de los tipos de CD, en la que se observa que en el grupo control la competencia reflexiva y actitudinal fue la más elevada con un promedio de 0,37 (medio bajo desarrollo de acuerdo al baremo de conversión), mientras que la competencia cognoscitiva fue la más baja $(0,22)$ en promedio la dimensión alcanzó un bajo desarrollo de competencias digitales $(0,29)$. En el caso del grupo experimental, la media más elevada fue 0,32 tanto para las competencias cognoscitivas como para las reflexivas y actitudinales, ambas califican de acuerdo con el baremo establecido (tabla 2) en la categoría de bajo desarrollo, y la competencia procedimental la media fue de 0,20, la cual también se categoriza como de bajo desarrollo. Los resultados en el grupo experimental lo sitúan en la categoría de bajo desarrollo de las $\mathrm{CD}$, con una media aritmética de 0,28.

En este sentido, se pudo diagnosticar que ambos grupos (grupo control y grupo experimental), presentan un bajo desarrollo en las CD tanto cognoscitivas, procedimentales como las reflexiva y actitudinales, que además no existen diferencias entre los grupos, siendo necesaria la aplicación de un diseño en uno de los grupos, en este caso el que se determinó grupo experimental, con el propósito de examinar la efectividad de los videos didácticos para el desarrollo de las CD.

Tabla 6

Desarrollo de los tipos de competencias D (Post prueba)

\begin{tabular}{|l|c|c|c|}
\hline Indicadores & Cognoscitivos & Procedimentales & Reflexivos y actitudinales \\
\hline Grupo control & $\begin{array}{c}0,40 \\
0,40\end{array}$ \\
\hline Promedio de la dimensión & Medio bajo desarrollo & Bajo desarrollo & Medio bajo desarrollo \\
\hline Grupo experimental & $\begin{array}{c}0,74 \\
0,33 \text { (Bajo desarrollo) }\end{array}$ \\
\hline Promedio de la dimensión & Alto desarrollo & 0,46 & $\begin{array}{c}0,48 \\
\text { Medio bajo desarrollo }\end{array}$ \\
\hline
\end{tabular}

Fuente: elaboración propia

Finalmente, la tabla 6, presenta los resultados comparativos de la post prueba aplicada a ambos grupos, donde se observó que para el grupo experimental el mayor incremento de la media aritmética ocurrió en las competencias cognoscitivas $(0,74)$ es decir, se ubicó en la categoría de alto desarrollo. Mientras que, la media alcanzada por el indicador de las competencias procedimentales solo alcanzó el 0,46 es decir, un medio bajo desarrollo de la competencia en los estudiantes. Estos resultados permitieron que la media general de la 
dimensión se movilizara de categoría cuantitativa con respecto al pretest, ubicándose en 0,56 es decir, medio alto desarrollo de las competencias digitales.

En cambio, el grupo control luego de transcurrido el mismo tiempo, pero sin el uso del diseño que empleó el grupo experimental, registró leves movilizaciones en su desempeño al aplicar el postest. Se destaca que, si bien las competencias cognoscitivas, reflexivas y actitudinales representaron un avance en el nivel de desarrollo ubicándose cualitativamente en el nivel medio bajo en ambos casos, esto no sugiere condiciones favorables en el actual contexto de la sociedad y los avances educativos. De hecho, el indicador menos favorable es justamente el relacionado con el saber-hacer, es decir, usar, que no se movió de categoría, pues se mantuvo en bajo desarrollo de las $C D$, tal como en el pretest.

\section{Conclusiones}

Tomando en cuenta los resultados anteriormente presentados, con la finalidad de evaluar el uso de los vídeos didácticos para el fortalecimiento de las competencias digitales en los estudiantes del grado décimo de la Institución Educativa Distrital Técnico Industrial de Santa Marta, Colombia, al diagnosticar los tipos de competencias digitales, se pudo determinar que los estudiantes presentaron un bajo desarrollo de las competencias en ambos grupos (control y experimental), donde todas presentaron valores bajos especialmente las competencias digitales cognitivas, por su parte, las reflexivas y actitudinales se ubicaron ligeramente en valor más alto, pero igual dentro del rango deficiente, siendo necesario el desarrollo de las mismas. Seguidamente, se elaboró el diseño instruccional para el uso de vídeos didácticos para el desarrollo de CD en los estudiantes, tomando en cuenta modelos existentes.

Al determinar el desarrollo de los tipos de CD en los estudiantes, se puede concluir que los valores obtenidos se mantuvieron casi al mismo nivel previo a aplicar las clases tradicionales, presentándose incipientes desarrollos, mostrándose los valores más significativos en las competencias digitales reflexivas y actitudinales, llevando a un mediano desarrollo, mientras que las otras se mantienen en niveles bajos.

Finalmente, al comparar el desarrollo de los tipos de CD en los estudiantes del grado décimo que recibieron clases de manera tradicional con los del grupo que se les aplicó el diseño instruccional para el uso de vídeos didácticos

Se concluye que es efectivo el uso de videos didácticos para el desarrollo de competencias digitales, sin embargo, se requiere seguir trabajando en su incidencia en las competencias digitales procedimentales, reflexivas y actitudinales, ya que en ninguno de los casos el desempeño alcanzó los máximos niveles.

Se recomienda emplear los videos didácticos en el proceso de cualificación, la creación a comunidades de aprendizaje, el manejo de herramientas tecnológicas que permiten desarrollar eficientemente procesos educativos, a fin de que los mismos diversifiquen sus prácticas logrando obtener mejores resultados y alcance de los objetivos trazados. Adicionalmente compartan y se apoyen en la construcción del conocimiento, evitando competir. Solo de esta manera, se verá evidenciado en mejores prácticas y con ello mejores procesos educativos, tecnológicos y sociales. Finalmente, crear y compartir este tipo de recursos para el trabajo eficiente con la diversidad según sea sus características apoyarse en sus bondades para el trabajo.

\section{Declaración de conflictos}

Para la redacción del presente artículo de investigación se manifiesta que los autores no tienen intereses ajenos a publicar y dejan constancia no han incurrido en actuaciones indebidas para lograr tal fin. 


\section{Referencias bibliográficas}

Alvarado, A.; Dehesa, J.; López, E. y Márquez, J. (2017). Los vídeos tutoriales como apoyo al proceso de enseñanza aprendizaje y sus implicaciones pedagógicas en el diseño instruccional. Educateconciencia, 14(15), 67-86.

Arango, D. A. G., Villa, C. F. H., Rojas, Ó. A. C., Fernández, J. E. V., \& Gutiérrez, C. A. E. (2020) Caracterización competencias digitales en docentes universitarios: estudio de caso1. Ph. D.(C) Diego Alejandro Correa Correa Mg. Carlos Mario Correa Cadavid, 91.

Bernal-Torres, C. A., Hoyos, J. D., Fracica, G., \& Fernández-Otoya, F. A. (2020). Uso del video en el aprendizaje del taekwondo: estudio en niños en edad escolar. Información tecnológica, 31(1), 311-320.

Benítez Larghi, Sebastián (2020). La construcción de habilidades digitales estudiantiles en torno al Programa Conectar Igualdad. Ciencia, Docencia y Tecnología, 31(60),131-154

Bolaño, M. (2019). Modelo basado en el uso de las TIC para la inclusión de estudiantes con capacidades diversas. Didáctica, innovación y multimedia. (37), S/P. Recuperado:

https://www.raco.cat/index.php/DIM/article/viewFile/356941/448869

Cejas Martínez Magda, Nohemi, Lozada Arias Brenda, Jacqueline, Urrego Ana, José, Mendoza Velazco Derling, \& Gabriela, Rivas Urrego. (2020). La irrupción de las tecnologías de la información y la comunicación (TIC), un reto en la gestión de las competencias digitales de los profesores universitarios en el Ecuador. RISTI Revista Ibérica de Sistemas e Tecnologias de Informação, (37), 132-

148. https://dx.doi.org/10.17013/risti.37.131-148

Espinoza-Freire, E. E. (2020). Políticas educativas de integración de las tecnologías de la información y las comunicaciones en el sistema educativo. Maestro y Sociedad, 17(1), 52-63.

Fernández-Cruz, F., \& Fernández-Díaz, M. (2016). Generation Z's teachers and their digital skills. [Los docentes de la generación Z y sus competencias digitales]. Comunicar, 46, 97-105. https://doi.org/10.3916/C462016-10

Gálvez, I. E. (2017). La UNESCO. Educación en todos los sentidos. Revista Complutense de Educación, 28(1), 345.

Gálvez Díaz, M. D. P., \& Tolaba, C. (2019). Como alcanzar competencias usando Scrum. In I Simposio Argentino de Educación en Informática (SAEI 2019)-JAllO 48 (Salta).

González, O. (2018). El video tutorial como herramienta de educación no formal en estudiantes de Bogotá, Colombia. Question/Cuestión, 1(59), e071-e071.

Instituto Nacional de Evaluación Educativa. (2016). Revisión de la OCDE de las políticas para mejorar la efectividad del uso de los recursos educativos, Informe país. S/N. Recuperado de: http://www.oecd.org/education/school/CBR\%20Spain\%20Spanish\%20version.pdf

Kerlinger, F. N y Lee, H. B (1999). Investigación del comportamiento. California, UEA: McGraw-Hill.

Leal Urueña, L. A. (2020). La formación inicial en competencias digitales del profesorado de Secundaria: una lectura desde las ecologías de aprendizaje. Recuperado de http://espacio.uned.es/fez/eserv/tesisuned:ED-Pg-Educac-Laleal/LEAL_URUENA_LINDA_Tesis.pdf 
Levano-Francia, Luz, Sanchez Diaz, Sebastian, Guillén-Aparicio, Patricia, Tello-Cabello, Sara, Herrera-Paico, Nancy, \& Collantes-Inga, Zoila. (2019). Digital Competences and Education. Propósitos y Representaciones, 7(2), 569-588. https://dx.doi.org/10.20511/pyr2019.v7n2.329

Lizana Carrió, A. (2018). Recursos tecnológicos en educación

Linarez, G. (2015). Los vídeo-tutoriales en la educación universitaria del siglo XXI. Revista Iberoamericana de Producción Académica y Gestión Educativa. 2, 1-11. Recuperado de https://www.pag.org.mx/index.php/PAG/article/download/463/502.

Márquez, A. X. H., Espinosa, J. L. S., Barrera, F. L., \& Cervantes, P. P.(2020) The development of digital teaching resources for learning in Biological Health, Behavioral and Chemical Sciences/El desarrollo de recursos didácticos digitales para el aprendizaje en Ciencias de la Salud, el Comportamiento y Químico Biológicas. Red de Investigación en Salud en el Trabajo, 3(4), 11-24. Recuperado de https://rist.zaragoza.unam.mx/index.php/rist/article/view/84/169

Mañas Pérez, A., \& Roig-Vila, R. (2019). Las Tecnologías de la Información y la Comunicación en el ámbito educativo. Un tándem necesario en el contexto de la sociedad actual.

Ministerio de Educación Nacional. 2008. Guía para el mejoramiento institucional

Morales-Rocha, J., Maquera-Luque, P., \& Herrera-Córdova, F. (2020). Desarrollo de competencias investigativas a través del semillero de la escuela profesional de Gestión Pública. Dominio de las Ciencias, 6(3), 48-64. doi:http://dx.doi.org/10.23857/dc.v6i3.1383

Nieto Hernández, M., \& De la Rosa Vergara, B. D. (2019) Talleres de alfabetización digital surgidos a partir del programa UNAMITAi Ciérrale a la brecha digital!: Recuperado de http://unamitadigital.filos.unam.mx/wpcontent/uploads/2020/02/ponencia-educatic-2.pdf

Orellana, A., y Pósito, R. M. (2019, junio). Analíticas del aprendizaje en el uso de videos en el ámbito universitario. Ponencia presentada en el XXI Workshop de Investigadores en Ciencias de la Computación. Puerto Rico.

Paz Maldonado, E. J. (2018). La formación del profesorado universitario para la atención a la diversidad en la educación superior. IE Revista de investigación educativa de la REDIECH, 9(16), 67-82.

Perdomo, B., Martinez, O. G., \& Barreto, I. B. (2020). Competencias digitales en docentes universitarios: una revisión sistemática de la literatura. EDMETIC, 9(2), 92-115. Recuperado de https://upo.es/revistas/index.php/IJERI/article/view/4387/4435

Ruiz E., Ruiz G., \& Odstrcil, M. (2007). Metodología para realizar el seguimiento académico de alumnos universitarios. Revista Iberoamericana de Educación, 42(3), 2-9.

Robles, C., y Zambrano, L. (2020). Prácticas académicas basadas en las nuevas tecnologías para el desarrollo de ambientes creativos de aprendizaje. Rehuso, 5(2), 50- 61. Recuperado de: https://revistas.utm.edu.ec/index.php/Rehuso/article/view/1684

Troncoso-Pantoja, Claudia A, Díaz-Aedo, Fredy, Amaya-Placencia, Juan P, \& Pincheira-Aguilera, Susana. (2019). Elaboración de videos didácticos: un espacio para el aprendizaje activo. FEM: Revista de la Fundación Educación Médica, 22(2), 91-92. Recuperado en 21 de octubre de 2020, de http://scielo.isciii.es/scielo.php?script=sci_arttext\&pid=S2014-98322019000200007\&lng=es\&tlng=es.

UNESCO (1997). Primer Estudio Regional Comparativo y Explicativo (PERCE). Santiago de Chile: UNESCO 
Vargas, E. (2020). La educación tecnocientífica en América Latina. AULA Revista de Humanidades y Ciencias

Sociales, 66(1), 21-28.Recuperado de https://revistas.unphu.edu.do/index.php/aula/article/view/124/124

Velázquez, A. K. O., \& Cupil, R. G. (2020). LA RESIGNIFICACIÓN PROFESIONAL A TRAVÉS DE LA FORMACIÓN EN COMPETENCIAS DIGITALES. Presencia Universitaria, (12), 74-85.

Vygotsky, L. S. (1977). The development of higher psychological functions. Soviet Psychology, 15(3), 60-73.

Esta obra está bajo una Licencia Creative Commons

Attribución-NoCommercial 4.0 International

(cc) BY-NC 